\title{
Coupled Radiation Effects in Thermochemical Nonequilibrium Shock-Capturing Flowfield Calculations
}

\author{
Lin C. Hartung, ${ }^{*}$ Robert A. Mitcheltree, and Peter A. Gnoffo ${ }^{\dagger}$ \\ NASA Langley Research Center,
}

\begin{abstract}
Lunar and Mars return conditions are examined using the LAURA flowfield code and the LORAN radiation code to assess the effect of radiative coupling on axisymmetric thermochemical nonequilibrium flows. Coupling of the two codes is achieved iteratively. Special treatment required to couple radiation in a shock-capturing method is discussed. Results indicate that while coupling effects are generally the same as occur in equilibrium flows, under certain conditions radiation can modify the chemical kinetics of a nonequilibrium flow and thus alter relaxation processes. Coupling effects are found to be small for all cases considered, except for a five meter diameter aerobrake returning from Mars at $13.6 \mathrm{~km} / \mathrm{sec}$.

\section{Nomenclature}

c Speed, $\mathrm{cm} / \mathrm{sec}$

\footnotetext{
*Aerospace Engineers, Aerothermodynamics Branch, Space Systems Division, NASA Langley Research Center, Hampton, VA 23681-0001, Members AIAA.

${ }^{\dagger}$ Aerospace Engineer, Aerothermodynamics Branch, Space Systems Division, NASA Langley Research Center, Hampton, VA 23681-0001, Associate Fellow AIAA.

Received June 10, 1993; presented as Paper 92-2868 at the AIAA 27th Thermophysics Conference, Nashville, TN, July 6-8, 1992; revision received Aug. 13, 1993; accepted for publication Aug. 25, 1993. Copyright (c) 1993 by the American Institute of Aeronautics and Astronautics, Inc. No copyright is asserted in the United States under Title 17, U.S. Code. The U.S Government has a royalty-free license to exercise all rights under the copyright claimed herein for Governmental purposes. All other rights are reserved by the copyright owner.
} 


$\begin{array}{ll}d_{q} & \text { Radiative flux relaxation parameter } \\ N & \text { Number density, } \mathrm{cm}^{-3} \\ q & \text { Heat flux, } \mathrm{W} / \mathrm{cm}^{2} \\ s & \text { Path length, } \mathrm{cm} \\ T & \text { Temperature, } \mathrm{K} \\ V_{\infty} & \text { Freestream velocity, } \mathrm{km} / \mathrm{sec} \\ z & \text { Stagnation line coordinate, } \mathrm{cm} \\ \eta & \text { Normal coordinate, } \mathrm{cm} \\ \rho_{\infty} & \text { Freestream density, } \mathrm{kg} / \mathrm{m}^{3} \\ \sigma & \text { Cross-section, } \mathrm{cm}{ }^{2} \\ \tau & \text { Relaxation time, } \mathrm{sec}\end{array}$

\section{Sub- and Superscripts}

$\begin{array}{ll}e & \text { electron } \\ M W & \text { Millikan and White } \\ P & \text { iteration level } \\ \mathrm{R} & \text { Park } \\ s & \text { radiative } \\ t & \text { Sound } \\ v & \text { translational } \\ & \text { vibrational }\end{array}$

\section{Introduction}

A blunt-nosed vehicle traveling through a gaseous medium at hypersonic speed may experience significant radiative heating. This heating results from radiative energy emitted in the shock layer without directional preference. That portion of energy which is radiated 
away from the vehicle and escapes the shock layer causes the phenomenon called radiative cooling. So far, flows with significant radiative cooling have been simulated only by fixedshock schemes including the viscous-shock-layer (VSL) scheme ${ }^{1-4}$ or inviscid equilibrium schemes $^{5}$. This approximation simplifies the calculation of the coupled, radiating flowfield but may not be valid for flow situations characteristic of aerobraking reentry into the Earth's atmosphere upon return from the Moon or Mars.

The objective of the present work is to examine radiative coupling effects in shockcapturing, thermochemical nonequilibrium flowfield calculations. A number of freestream conditions, representative of Lunar and Mars return proposals, are studied. The Langley Optimized RAdiative Nonequilibrium (LORAN) $\operatorname{code}^{6,7}$, is coupled to the Langley Aerothermodynamic Upwind Relaxation Algorithm (LAURA) flowfield solver for this purpose. LORAN includes options for both tangent slab and an approximate 3 -D radiation transport calculation. Results presented in this paper utilize the tangent slab approximation. LAURA is a shock-capturing Navier Stokes solver which includes 11-species chemical nonequilibrium and the two-temperature thermal nonequilibrium model of Park $^{8}$. Coupling of the LAURA and LORAN models is achieved through an iterative procedure, in which the radiation properties are updated every 200 to 5000 flowfield iterations, depending on the flow conditions. Special treatment of the shock and shock precursor regions is found to be necessary and is described here. Additional numerical considerations are also necessary to establish a convergent, efficient scheme.

The coupled LAURA/LORAN code has been applied to a range of axisymmetric flowfields representative of missions of current interest. The effects of radiation coupling in a shock-capturing scheme for thermochemical nonequilibrium flowfields with significant ionization are found to differ from those observed in equilibrium or near-equilibrium flowfields. In particular, radiative cooling can alter the ionization chemistry in the flowfield, resulting in an increase in translational temperature and shock standoff distance. 


\section{Radiation Coupling Theory}

Radiation is inherently coupled to the state of the gas from which it emanates. It results from radiative transitions in the internal energy states of the atoms, molecules and ions present in the gas. The radiation emitted by such transitions travels through the gas at the speed of light until it is reabsorbed or exits the flowfield. Radiation thus provides an additional energy transport mode. Radiation can also affect the chemical state of the gas through phenomena such as photodissociation, photoionization, and radiative recombination.

Energy transport through the gas by radiation is modeled in the Navier-Stokes equations with a term which appears in the energy equation: the divergence of the radiative flux, $\nabla \cdot \vec{q}_{\mathrm{R}}$ (denoted $Q_{\text {rad }}$ by Gnoffo $^{9}$ ). This divergence term is computed in the LORAN radiation-transport model. When the tangent slab approximation is used, $\nabla \cdot \vec{q}_{\mathrm{R}}=d q_{\mathrm{R}} / d \eta$. The radiation effects on chemical kinetics can be included by adding photo-reactions to the chemistry model of the gas and accounting for their effect on the radiation properties. The radiation effects on chemical kinetics are not included in the current LAURA/LORAN code. This approximation worsens as the energy in the flow increases, but has not been quantitatively assessed.

The transport of energy due to radiation is usually neglected in the aerothermodynamic analysis of hypersonic flowfields. For flowfields where this assumption is not valid, the effects of radiation can be determined by comparing solutions from calculations with and without radiative transport. In general, the shock layer becomes non-adiabatic when radiation transport is included. The enthalpy of a parcel of fluid decreases as it traverses the layer from the shock to the body due to radiative cooling. For equilibrium gas calculations, the resulting temperature profiles are no longer constant but decrease monotonically across the layer. Lower average temperatures result in higher average densities and thus decreased shock standoff distance. The combination of lower temperatures and a thinner shock layer reduces the radiative heat load to the wall.

In a thermochemical nonequilibrium gas calculation, multiple temperatures are defined 
to describe the partitioning of energy across the internal modes. The number of temperatures required to adequately describe the flow depends on the conditions. For example, rotational, vibrational, and electronic energy modes for the mixture or for each species may be used. The various states of each energy mode are assumed to be in equilibrium at that mode's characteristic temperature. Each mode is described by its own energy conservation equation which includes terms coupling it to the other energy modes. Usually, separate internal modes for each species are not defined since the energy modes are strongly coupled between species.

To correctly model the radiative coupling in the nonequilibrium case only that portion of $\nabla \cdot \vec{q}_{\mathrm{R}}$ associated with transitions of each particular energy mode should be included in the energy equation for that mode. Since purely rotational radiative transitions occur at low infrared energies - which are generally neglected in gas radiation — it may be assumed that all radiative transitions of interest in air involve a transition between vibrational or electronic states. LAURA employs the two-temperature model ${ }^{8}$, requiring a total energy equation $\left(T_{t}\right)$ and a combined vibrational - electron - electronic energy equation $\left(T_{v}\right)$. Thus the complete $\nabla \cdot \vec{q}_{\mathrm{R}}$ appears in both energy equations.

\section{Numerical Algorithm}

The LAURA algorithm for solution of the Navier Stokes equations is summarized in Ref. 10. The divergence of the radiative flux can be treated as a source term in the two energy equations. In general, source terms are handled implicitly in the LAURA algorithm. This treatment improves the scheme's stability and robustness. Since radiation is sensitive to flowfield properties, it would be advantageous to treat the added radiation source term implicitly also. Park and Milos ${ }^{11}$, however, have found this implicit treatment to be prohibitively expensive (in computer time) for anything except 1-D flows. Furthermore, it is not clear how to obtain the necessary terms for the Jacobian in the current nonequilibrium treatment. The radiation term is therefore treated as an explicit source term in the LAURA algorithm. 
The convergence of the flowfield in LAURA is characterized by transient waves in the gas properties. When the sensitive radiation calculation is performed on a flowfield still containing transients, the radiation term can change dramatically. This can introduce additional fluctuations and lead to instability. In the present study, therefore, under-relaxation is applied to the explicit radiation source term. That is, the latest radiation solution is combined with the previous solution to compute an updated radiation term:

$$
\left(\nabla \cdot \vec{q}_{\mathrm{R}}\right)^{n^{\prime}+1}=d_{q}\left(\nabla \cdot \vec{q}_{\mathrm{R}}\right)^{n}+\left(1-d_{q}\right)\left(\nabla \cdot \vec{q}_{\mathrm{R}}\right)^{n+1}
$$

The under-relaxation approach of Eq. 1 reduces the fluctuations due to coupling and increases the stability of the combined algorithm. A relaxation factor, $d_{q}$, of 0.5 is used for the results presented.

Because the flow properties change little in a single flowfield iteration, and because it is advantageous to allow flowfield transients to decay, it is not necessary to update the radiative flux divergence term every time the flowfield is updated. In fact, the total cost of the combined computation can be reduced several orders of magnitude by updating the radiation term a minimum number of times, as is successfully done in equilibrium shockfitting methods. Not updating it frequently enough, however, can decrease the convergence rate, produce oscillatory non-convergent behavior, or destabilize the solution. For example, if the flowfield changes dramatically between radiation term updates, a large change in the radiation will result and can introduce severe waves into the flowfield. In addition, if flow structures change position significantly between updates of the radiation, the radiation term will temporarily not be "aligned" with the associated flow structure. Experience with cases characterized by a moderate degree of nonequilibrium suggests that updating the radiation calculation once for every 500 flowfield updates provides reasonable convergence. For cases with more severe nonequilibrium in which coupling has a large effect on the standoff distance, more frequent updates are required (every 200 iterations). As a flowfield approaches equilibrium, on the other hand, the current LAURA algorithm converges more slowly. For near-equilibrium cases, the radiation is updated only every 1000 to 5000 flowfield iterations. 
Obtaining coupled solutions may be difficult when extreme gradients occur in the shock wave, as is the case for near-equilibrium flows. The computational mesh used in a flowfield calculation seldom provides adequate resolution in the shock region to produce smooth temperature profiles in such flows. This lack of resolution at the shock does not significantly degrade the flowfield solution. Since the radiation calculation is very sensitive to temperature, however, it can cause large changes in the profile of $d q_{\mathrm{R}} / d \eta$ which may lead to inaccurate results or even divergent solutions. Increasing the number of grid points can alleviate this problem but is not always practical because of storage limitations. Grid adaption, on the other hand, can improve the temperature profiles without increased storage. To provide this ability, a grid adaption algorithm has been added to the LAURA code. In this study it is used to cluster grid points in the vicinity of the maximum temperature in the shock.

The low-temperature, or precursor, region ahead of the shock in a shock-capturing scheme also requires special treatment. The LORAN radiation model as currently implemented does not include accurate radiation properties at low temperatures. It is generally assumed that the precursor has a negligible effect on the converged flowfield, since it can absorb only a small fraction of the radiative energy passing through it. Recent results ${ }^{12}$ confirm the validity of this approximation. Therefore, $d q_{\mathrm{R}} / d \eta$ is set to zero - the value appropriate for a non-participating medium - when either temperature (vibrational or translational) is below a certain minimum. This is done within the LAURA flowfield calculation every iteration. Even if accurate precursor (low - temperature) radiation properties are available, some logic is still required so that any decrease in shock standoff distance which might occur between radiation updates will not leave a large radiation term in what is now the freestream. The value used for this minimum temperature is $500 \mathrm{~K}$ for the cases in this paper.

\section{Physical Models in LAURA}

The complete set of physical models used in the LAURA code is described in Ref. 9. Options exist for some of these models and a number of parametric studies have been performed 
to assess the differences between them ${ }^{13-16}$. For the results presented here, LAURA utilizes the Park-91 chemical kinetics model ${ }^{17}$ (except for the Mars capsule return case which uses the Park- 87 model $\left.^{8}\right)$. The dissociation rate controlling temperature is modeled by $\sqrt{T_{t} T_{v}}$ for the LTV and Mars aerobrake cases, and by $T_{t}^{7} T_{v}^{3}$ for the capsule return cases. The vibrational - translational relaxation time is modeled as

$$
\tau_{v}=\tau_{v}^{M W}+\tau_{v}^{P}
$$

with

$$
\tau_{v}^{P}=\left(\sigma_{v} \overline{c_{s}} N\right)^{-1}
$$

where the effective cross section for vibrational relaxation is

$$
\sigma_{v}=10^{-17}\left(50,000 / T_{t}\right)^{2} \mathrm{~cm}^{2}
$$

and $\tau_{v}^{P}$ is calculated with the total number density. Relative to results presented earlier ${ }^{13}$, this causes faster relaxation of the vibrational energy mode (as was suggested in Ref. 13 by comparison to the FIRE II results). The vibrational energy removed in dissociation is assumed to be the average vibrational energy, while in ionization it is assumed to be the energy to ionize from the ground state.

The wall boundary condition for the LTV and Mars aerobrake cases is Stewart's finite wall catalysis model ${ }^{18}$. The capsule return cases employ the "super-catalytic" boundary condition in which freestream species concentrations are enforced at the wall.

While these models have been validated to the extent possible, using FIRE II flight data and ground-based experimental results, they nevertheless retain significant uncertainties as does the radiation model. Their application to the Mars return conditions represents an extrapolation from any known data. The results presented below should be viewed as presenting trends rather than absolute heating levels.

\section{Results}

The effects of radiation coupling on thermochemical nonequilibrium flows are illustrated 
by comparing uncoupled and coupled solutions from the LAURA/LORAN code. An uncoupled wall radiative flux is computed by applying the LORAN code to the uncoupled LAURA output. Flow conditions representative of aerobraking reentry of axisymmetric sphere cone bodies into the Earth's atmosphere upon return from the Moon and Mars are examined.

\section{Lunar Return Conditions}

Two lunar return conditions are studied. The first is a small body representative of a capsule return. The peak convective heating point on this trajectory at $9.871 \mathrm{~km} / \mathrm{sec}$ and $75 \mathrm{~km}$ altitude $\left(\rho_{\infty}=4.623 \times 10^{-5} \mathrm{~kg} / \mathrm{m}^{3}\right)$ is examined. A 42 by 64 cell grid defines the forebody of this $3 \mathrm{~m}$ nose radius, $70^{\circ}$ sphere cone capsule. The second case is representative of a large lunar transfer vehicle (LTV). The peak convective heating point at $9.797 \mathrm{~km} / \mathrm{sec}$ and $72 \mathrm{~km}$ altitude $\left(\rho_{\infty}=5.987 \times 10^{-5} \mathrm{~kg} / \mathrm{m}^{3}\right)$ is computed. A 30 by 64 cell grid defines the forebody of this spherical aerobrake which is $15.24 \mathrm{~m}$ in diameter, with a $13.58 \mathrm{~m}$ nose radius and a $0.3048 \mathrm{~m}$ radius shoulder.

\section{Lunar Capsule Return}

The stagnation line temperature profiles from the uncoupled solution for the capsule return case are shown by the solid lines in Fig. 1. Thermal nonequilibrium effects are restricted to about 20 percent of the shock layer in this near-equilibrium case. The dashed lines in the figure are the temperature profiles from the coupled solution. For this nearequilibrium case, the behavior of the coupled solution is the same as has been observed with equilibrium flowfields: the radiation cools the flow, leading to lower temperatures (which do not level off to an equilibrium value as in the uncoupled solution), higher density, and thus a smaller shock standoff distance. The result is a temperature which is 200 to $250 \mathrm{~K}$ lower through much of the shock layer. There is no detectable effect on the vibrational relaxation distance: the temperatures relax to thermal equilibrium in about $3 \mathrm{~cm}$ whether coupling is included or not.

The effect of coupling on the surface heating contributions is shown in Fig. 2. Radiative 
cooling results in lower surface radiative heating all along the body. At the stagnation point the coupled result is 7 percent lower. It is 20 percent lower on the body flank, where the volume of radiating gas in the shock layer is reduced the most for this sphere cone geometry. The effect on convective heating is small. It decreases a maximum of three percent at the stagnation point. This insensitivity is expected because of the competing effects of lower shock layer temperatures, boundary layer absorption of radiation, and thinning of the shock layer.

For the near-equilibrium capsule return case, starting from a converged uncoupled solution, about 22000 flowfield iterations with eight radiation updates were required to achieve the converged coupled solution. This required about eight hours of Cray-2 CPU time. The reason for this large computer time is the very near-equilibrium character of this case. In order to provide a smooth solution for the wall radiative heat flux, the temperature gradients in the shock must be resolved to a much greater accuracy than is required for a reasonable flowfield solution. For this case, the necessary resolution was achieved through a trial and error succession of grid adaptions, convergence runs, and radiation updates. An optimal selection of the adaption and radiation update intervals would have required less CPU time.

\section{Lunar Transfer Vehicle}

The stagnation line temperature profiles for the LTV are shown in Fig. 3. This case is very near equilibrium, with thermal relaxation occurring in the outer 7 percent of the shock layer. The effect of radiative coupling is small. It reduces the shock standoff distance by about 4 percent and produces continually decreasing temperatures through the shock layer. The temperatures are 300 to $400 \mathrm{~K}$ lower over a substantial part of the shock layer. These trends are again the same as for equilibrium.

The effect on the surface heating for the LTV is shown in Fig. 4. For this spherical forebody, the radiative flux decreases monotonically away from the stagnation point. The radiative heat flux is reduced 15 to 20 percent by coupling, while the convective heating 
decreases 7 to 9 percent. The larger radiative flux for this large body results in increased radiative cooling effects.

For this near-equilibrium case, starting from a converged uncoupled solution, about 22000 flowfield iterations were again required to obtain the coupled solution. Four radiation updates were performed. Fewer radiation updates were required for this case because the shock moved very little with coupling. The calculations required just over three hours of Cray Y-MP CPU time for this smaller grid.

\section{Mars Return Conditions}

Four Mars return conditions are considered. The first is a small body representing a capsule return. The flight condition is $12 \mathrm{~km} / \mathrm{sec}$ and $80 \mathrm{~km}$ altitude $\left(\rho_{\infty}=1.999 \times 10^{-5}\right.$ $\mathrm{kg} / \mathrm{m}^{3}$ ) with a fine 30 by 128 cell grid covering only the nose region of a $1.08 \mathrm{~m}$ nose radius, $60^{\circ}$ sphere cone. This case provides a detailed look at the stagnation region of this flowfield. The remaining three cases involve a geometry and conditions typical of an aerobraking reentry into the Earth's atmosphere ${ }^{19}$. The geometry is a scaled down version of the LTV which is 5 $\mathrm{m}$ in diameter with a $4.46 \mathrm{~m}$ nose radius and a $0.1 \mathrm{~m}$ radius shoulder. The grid is 30 by 64 cells. The three freestream conditions studied here span the entrance trajectory from near the continuum limit down to the predicted peak convective heating point.

\section{Mars Capsule Return}

The stagnation line temperature profiles from the uncoupled solution for the Mars capsule case are shown by the solid lines in Fig. 5. Half the shock layer is in thermal nonequilibrium. Temperature profiles for the coupled solution are also shown in Fig. 5 by the dashed lines. The effects of coupling are small but still affect the relaxation process in this nonequilibrium flow. While the vibrational temperature is again reduced by radiative cooling, the translational temperature increases in the nonequilibrium region. This unexpected behavior is attributed to thermal nonequilibrium effects in significantly ionized flows 
(25 percent ionization here vs 4 percent in the lunar cases). Examination of the electron number density profiles (Fig. 6) shows that the electrons are freed in two discernible surges: one immediately behind the shock due to the associative ionization reactions and one deeper in the shock layer due to electron impact ionization. The associative ionization reactions provide the free electrons necessary to initiate electron impact ionization. Since the vibrational temperature in this region is reduced as much as $800 \mathrm{~K}$ by coupling, both types of ionization are slowed. As a result the electron number densities are up to 75 percent different in the region of maximum coupling effects, and the maximum number density of electrons in the shock layer is reduced by 12 percent. The result is that the translational temperature remains higher (less energy is converted by ionization). The density in the shock layer therefore remains lower in the nonequilibrium region. With the compensating effect of higher density near the wall, the net effect is no change in the shock stand-off distance.

Radiation cooling has a small effect on the wall heat fluxes for this condition as shown in Fig. 7. The radiative flux is 5 to 9 percent lower in the coupled case, while the convective heating is 6 to 10 percent lower. (Recall that only the nose region of this aerobrake flowfield is computed.)

The convergence of this relatively nonequilibrium case was much smoother than for the near-equilibrium cases. Starting from a converged uncoupled solution, it required 4250 LAURA iterations and five radiation updates. This was done in under two hours of Cray Y-MP CPU time. A significant savings in this case is that grid adaption is unnecessary to resolve this diffuse shock. Once the initial transients from radiation were absorbed in the solution, radiation was updated every 500 LAURA iterations. Examination of the convergence histories suggests that this is a near-optimal interval.

\section{Mars Aerobrake Return - Point 1}

To examine the behavior of thermochemical nonequilibrium coupled flows, a point early in the aerobraking trajectory is studied. The velocity is $13.604 \mathrm{~km} / \mathrm{sec}$ at $91.5 \mathrm{~km}$ altitude 
$\left(\rho_{\infty}=1.239 \times 10^{-6} \mathrm{~kg} / \mathrm{m}^{3}\right)$.

Stagnation line temperature profiles for this case are shown in Fig. 8. The very nonequilibrium character of this flow is evident; the temperatures are out of equilibrium through most of the shock layer. The coupled profiles (dashed lines) show qualitatively the same behavior observed for the Mars capsule case, but with more pronounced effects. Radiation cooling decreases the vibrational temperature enough to inhibit ionization reactions in this 25 percent ionized flow. This results in more energy staying in the translational energy mode and a longer relaxation time. (Dissociation of molecules is also inhibited by the lower vibrational temperature, but this has less impact on the flow because the number densities and energies of formation are lower than for the ionized species.) The density is lowered and the shock standoff distance increases. The electron number density profiles are shown in Fig. 9. They show that the electron avalanche due to electron impact ionization reactions is delayed by about $10 \mathrm{~cm}$ inside the shock layer. As a result the maximum electron number density in the shock layer is reduced by a factor of three from the uncoupled result and the ionization fraction is lowered to 20 percent.

The effect on surface heating is shown in Fig. 10. Though the radiative heat load is small, nonequilibrium coupling effects are strong enough to reduce it by a factor of two or more. The convective heating increases 35 to 70 percent with coupling, due to reabsorption of radiation in the boundary layer. This is indicated by the higher temperatures near the wall for the coupled solution (Fig. 8). Despite the increase in convective heating, the total wall heat flux is reduced 18 to 25 percent by radiation coupling effects. Furthermore, while the uncoupled solution predicts radiation to be twice as strong as convective heating, the coupled solution has convection as the dominant mode of heat input to the wall.

Convergence of the coupled result for this very nonequilibrium case required more frequent radiation updates due to the large motion of the shock and the strong coupling effects. Updating radiation every 500 to 1000 iterations was found to cause an oscillatory behavior which converged very slowly if at all. To obtain reasonable convergence rates, it was 
necessary to update radiation every 200 LAURA iterations. Including the early oscillatory problems, convergence of this case took 9000 LAURA iterations, with 19 radiation updates. The complete trial and error process took under four hours of Cray Y-MP CPU time.

\section{Mars Aerobrake Return - Point 2}

A point 30 seconds later in the trajectory is studied to investigate radiative coupling effects as equilibrium is approached. Here the velocity is $13.585 \mathrm{~km} / \mathrm{sec}$ and the free-stream density is about an order of magnitude higher at $1.721 \times 10^{-5} \mathrm{~kg} / \mathrm{m}^{3}(80.7 \mathrm{~km}$ altitude). The nonequilibrium region is about a quarter of the shock layer, as shown by the temperature profiles in Fig. 11. Coupling reduces the stagnation point shock standoff distance by about ten percent. The relaxation zone is not qualitatively affected, however, and remains about $5 \mathrm{~cm}$ long.

The effect of the cooling on the wall radiative flux is large, resulting in a 25 to 35 percent reduction as shown in Fig. 12. The convective heating is unaffected by radiative coupling.

Converging this coupled solution starting from the uncoupled flowfield required 9600 LAURA iterations with six radiation updates. For this nonequilibrium case, radiation was generally updated every 500 to 1000 LAURA iterations. This solution required just under two hours of Cray Y-MP CPU time.

\section{Mars Aerobrake Return - Point 3}

The peak convective heating point $\left(12.883 \mathrm{~km} / \mathrm{sec}, \rho_{\infty}=1.598 \times 10^{-4} \mathrm{~kg} / \mathrm{m}^{3}\right.$ at $65 \mathrm{~km}$ altitude) on the aerobraking trajectory is an equilibrium flowfield. The relaxation zone is thinner than that shown in Fig. 3. The nonequilibrium LAURA/LORAN code is not wellsuited to this equilibrium problem. This case can, however, be used to examine the accuracy of the nonequilibrium LORAN code at equilibrium conditions.

Radiation was computed for the converged uncoupled LAURA flowfield with nonequilibrium LORAN and with equilibrium LORAN. The equilibrium version of LORAN replaces 
the quasi-steady-state (QSS) ${ }^{20}$ excited state populations with Boltzmann distributions. The distributions of wall radiative heat flux are compared in Fig. 13. They differ by two to eight percent. For an independent comparison, coupled and uncoupled inviscid equilibrium stagnation point solutions from the method of Ref. 5 are also shown in this figure. The agreement between the three uncoupled solutions at the stagnation point verifies that the nonequilibrium LORAN code approaches the equilibrium limit. The coupled inviscid equilibrium stagnation point solution indicates the large extent of radiative cooling present in this flow.

\section{Summary of Results}

The results from these six cases can be summarized in a form previously used to estimate coupling effects in equilibrium flows. Fig. 14 shows the cooling factor, $q_{\text {coupled }} / q_{\text {uncoupled }}$, as a function of the uncoupled radiation energy fraction, $q_{\text {uncoupled }} /\left(\rho_{\infty} V_{\infty}^{3} / 2\right)$. The denominator in the latter quantity is a measure of the total energy in the freestream. The broad crosshatched region represents extensive calculations with the equilibrium method of Ref. 5. The six nonequilibrium cases calculated in the present work are overlaid on this plot as circles. While they do not fall entirely within the equilibrium band, the nonequilibrium cooling factors show general agreement with the previous equilibrium results.

\section{Discussion}

Both LAURA and LORAN are collections of numerous, often complicated, physical models. There are questions about the validity of some of these models at the conditions examined. In particular, LAURA is a continuum Navier-Stokes model which assumes a weakly ionized two-temperature gas. The Mars return cases include degrees of ionization as high as 25 percent, which may violate the weakly ionized assumption. Point 1 for the Mars aerobrake case is at $91.5 \mathrm{~km}$ altitude - near the edge of the continuum region. In LORAN, the QSS assumption is open to question in high gradient captured shocks. Furthermore, 
neglecting the influence of radiation on photodissociation and photoionization may not be valid at these extreme conditions.

The reported computational cost of the coupled calculations is high in part because of uncertainty in the optimal frequency for updating the radiation calculation. This optimal frequency is shown to be related to the degree of nonequilibrium in the flowfield. Experience will lead to insight into selecting this frequency and may reveal an automatic mechanism which will result in a more efficient combined LAURA/LORAN code.

The results presented in this paper use the tangent slab transport approximation. It has been noted before ${ }^{6}$ that the variation of $\nabla \cdot \vec{q}_{\mathrm{R}}$ obtained from the approximate $3-D$ transport solution is smoother than the variation of $d q_{\mathrm{R}} / d \eta$ obtained from the tangent slab model. This smoothness would eliminate the need for some of the numerical considerations described above but at present represents a significant increase in computational cost. Future work will examine coupling with 3 -D radiative transport.

The results suggest that thermochemical nonequilibrium coupled radiation effects are important only at very low density (i.e. high altitude), high velocity conditions. The trajectories of most Earth entry vehicles continue deeper into the atmosphere so that the high

altitude heating is of minimal importance compared to the heat loads encountered at the lower altitudes. If an aerobraking trajectory is proposed which dissipates its kinetic energy in the upper, rarefied regions of the atmosphere, significant radiative cooling effects must be expected.

\section{Conclusions}

The LORAN thermochemical nonequilibrium radiation code is coupled with the LAURA flowfield code and applied to flowfields characteristic of Earth entry upon return from the Moon and Mars. Coupled solutions are obtained using the tangent slab radiation transport model. Coupling a radiation calculation to a flowfield solution by an iterative procedure gives rise to potential instabilities. These are avoided by the use of under-relaxation. In 
addition, special treatment of the radiation is required in the vicinity of the shock and in the shock precursor region which is not necessary in shock-fitting methods.

The results of this study indicate that radiation coupling effects are small for the lunar return cases considered. For these near-equilibrium cases, coupling effects are similar to those predicted by equilibrium shock-fitting methods. While the combined nonequilibrium LAURA/LORAN code is not an efficient means of computing equilibrium flowfields, it is shown to produce the correct equilibrium limit. For the higher energy Mars return cases, both nonequilibrium and coupling effects are stronger. In these cases with significant ionization, radiation cooling can alter the ionization chemistry of the flow. This change in chemistry can produce increased translational temperatures and result in a larger shock standoff distance. These effects are unlike those observed at equilibrium or near-equilibrium conditions. Nevertheless, the cooling factors for all these cases show agreement with previous equilibrium experience.

\section{Acknowledgments}

The equilibrium cooling factor results were provided by Dr. Kenneth Sutton of the Aerothermodynamics Branch at NASA Langley Research Center.

\section{References}

1 Nicolet, W. E., “Advanced Methods for Calculating Radiation Transport in AblationProduct Contaminated Boundary Layers," NASA CR 1656, September 1970.

${ }^{2}$ Zoby, E. V., Sutton, K., Olstad, W. B., and Moss, J. N., "Approximate Inviscid Radiating Flowfield Analysis for Outer Planet Entry Probes," Outer Planet Entry Heating and Thermal Protection, edited by Raymond Viskanta, Vol. 64, Progress in Astronautics and Aeronautics, AIAA, New York, 1979, pp. 42-64.

${ }^{3}$ Carlson, L. A., and Gally, T. A., “Nonequilibrium Chemical and Radiation Coupling Phenomena in AOTV Flowfields," AIAA Paper 91-0569, Jan. 1991. 
${ }^{4}$ Gupta, R. N., Lee, K. P., Moss, J. N., and Sutton, K., "A Viscous-Shock- Layer Analysis of the Martian Aerothermal Environment," AIAA Paper 91-1345, June, 1991.

5 Sutton, Kenneth, "Characteristics of Coupled Nongray Radiating Gas Flows with Ablation Products Effects About Blunt Blunt Bodies During Planetary Entries," PhD Thesis, North Carolina State University, Raleigh, North Carolina, 1973.

${ }^{6}$ Hartung, Lin C., "Nonequilibrium Radiative Heating Prediction Method for Aeroassist Flowfields with Coupling to Flowfield Solvers," PhD Thesis, North Carolina State University, Raleigh, North Carolina, 1991.

7 Hartung, Lin C., "Development of a Nonequilibrium Radiative Heating Prediction Method for Coupled Flowfield Solutions," AIAA Paper 91-1406, June 1991.

${ }^{8}$ Park, Chul, “Assessment of Two-Temperature Kinetic Model for Ionizing Air,” Journal of Thermophysics and Heat Transfer, Vol. 3, No. 3, 1989, pp. 233-244; also AIAA Paper 87-1574, June 1987.

9 Gnoffo, Peter A., Gupta, Roop N., and Shinn, Judy L., "Conservation Equations and Physical Models for Hypersonic Air Flows in Thermal and Chemical Nonequilibrium," NASA TP 2867, February 1989.

10 Gnoffo, Peter A., "Upwind-Biased Point-Implicit Relaxation Strategies for Viscous Hypersonic Flows," AIAA Paper 89-1972, June 1989.

11 Park, Chul, and Milos, Frank S., "Computational Equations for Radiating and Ablating Shock Layers," AIAA Paper 90-0356, January 1990.

12 Stanley, Scott A., and Carlson, Leland A., "The Effects of Shock Wave Precursors Ahead of Hypersonic Entry Vehicles," AIAA Paper 91-1465, June 1991.

13 Hartung, Lin C., Mitcheltree, Robert A., and Gnoffo, Peter A., "Stagnation Point Nonequilibrium Radiative Heating and the Influence of Energy Exchange Models," AIAA Paper 91-0571, January 1991.

14 Mitcheltree, Robert A., "Parametric Study of Dissociation and Ionization Models at 12 Kilometers/Second," Journal of Spacecraft \& Rockets, Vol. 28, No. 6, 1991, pp. 619-627; 
also AIAA Paper 91-1368, June 1991.

15 Greendyke, Robert B., Gnoffo, Peter A., and Lawrence, R. W., “Electron Number Density Profiles for the Aeroassist Flight Experiment," AIAA Paper 92-0804, Jan. 1992.

${ }^{16}$ Greendyke, Robert B., “A Parametric Analysis of Radiative Structure in Aerobrake Shock Layers," AIAA Paper 92-2970, July 1992.

17 Park, Chul, Howe, John T., Jaffe, Richard L., and Candler, Graham V., "ChemicalKinetic Problems of Future NASA Missions," AIAA Paper 91-0464, Jan. 1991.

18 Stewart, David A., Leiser, Daniel B., Kolodziej, Paul, and Smith, Marnell, “Thermal Response of Integral Multicomponent Composite Thermal Protection Systems," AIAA Paper 85-1056, 1985.

19 Braun, Robert D., Powell, Richard W., and Hartung, Lin C., "Effect of Interplanetary Trajectory Options on a Manned Mars Aerobrake Configuration," NASA TP 3019, Aug. 1990.

20 Park, Chul, “Nonequilibrium Hypersonic Aerothermodynamics," Wiley, New York, 1990. 


\section{Figure Captions}

1. Effect of radiation coupling on stagnation streamline temperature profiles for the lunar capsule

2. Effect of radiation coupling on surface heating for the lunar capsule

3. Effect of radiation coupling on stagnation streamline temperature profiles for the lunar transfer vehicle

4. Effect of radiation coupling on surface heating for the lunar transfer vehicle

5. Effect of radiation coupling on the stagnation streamline temperature profiles for the Mars capsule return condition

6. Effect of radiation coupling on stagnation streamline electron number density profiles for Mars capsule return condition

7. Effect of radiation coupling on surface heating for the Mars capsule return condition

8. Effect of radiation coupling on the stagnation streamline temperature profiles for the Mars aerobrake return condition - Point 1

9. Effect of radiation coupling on electron density for the Mars aerobrake return condition - Point 1

10. Effect of radiation coupling on surface heating for the Mars aerobrake return condition - Point 1

11. Effect of radiation coupling on the stagnation streamline temperature profiles for the Mars aerobrake return condition - Point 2

12. Effect of radiation coupling on surface heating for the Mars aerobrake return condition - Point 2

13. Wall radiative heating predictions for the Mars aerobrake return condition - Point 3

14. Cooling factor 


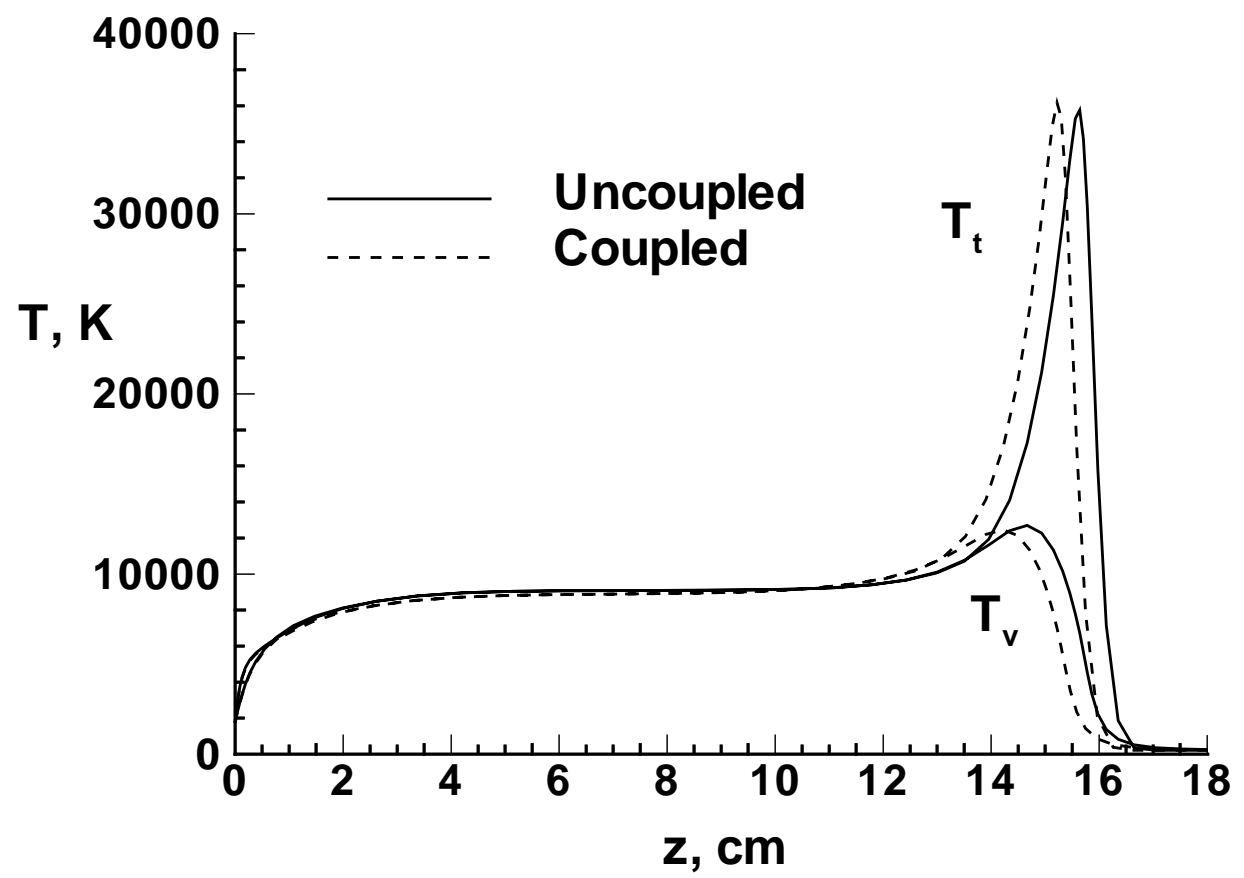

Figure 1: Effect of radiation coupling on stagnation streamline temperature profiles for the lunar capsule.

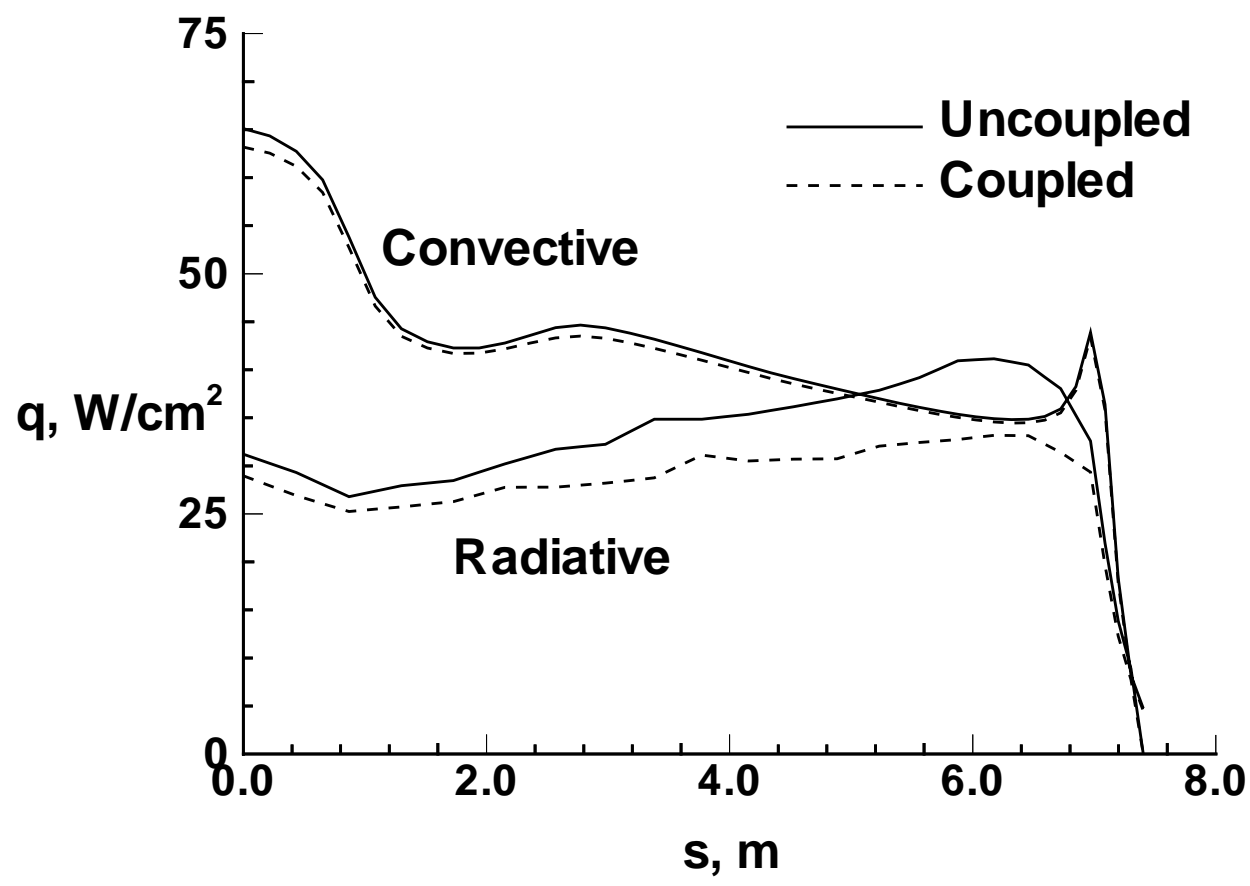

Figure 2: Effect of radiation coupling on surface heating for the lunar capsule. 


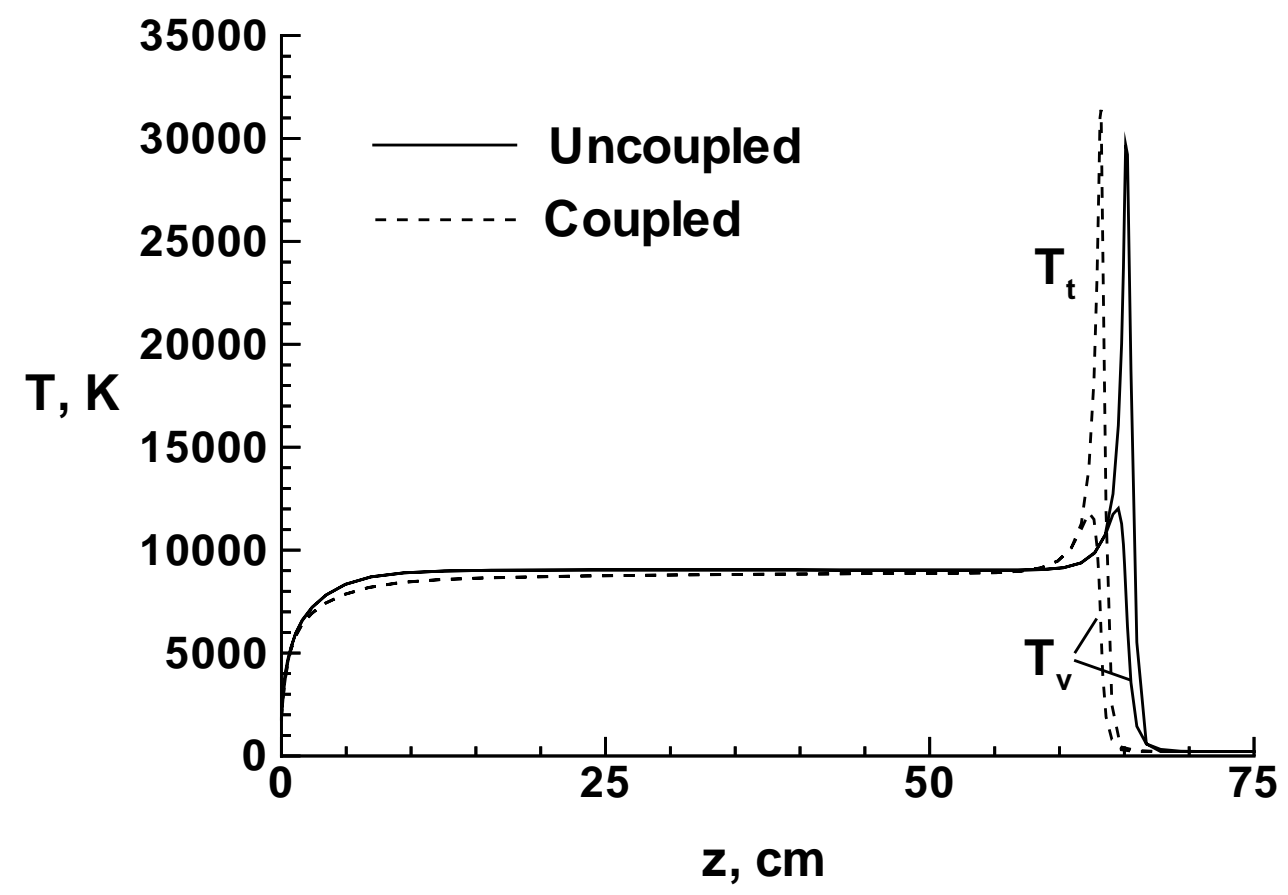

Figure 3: Effect of radiation coupling on stagnation streamline temperature profiles for the lunar transfer vehicle.

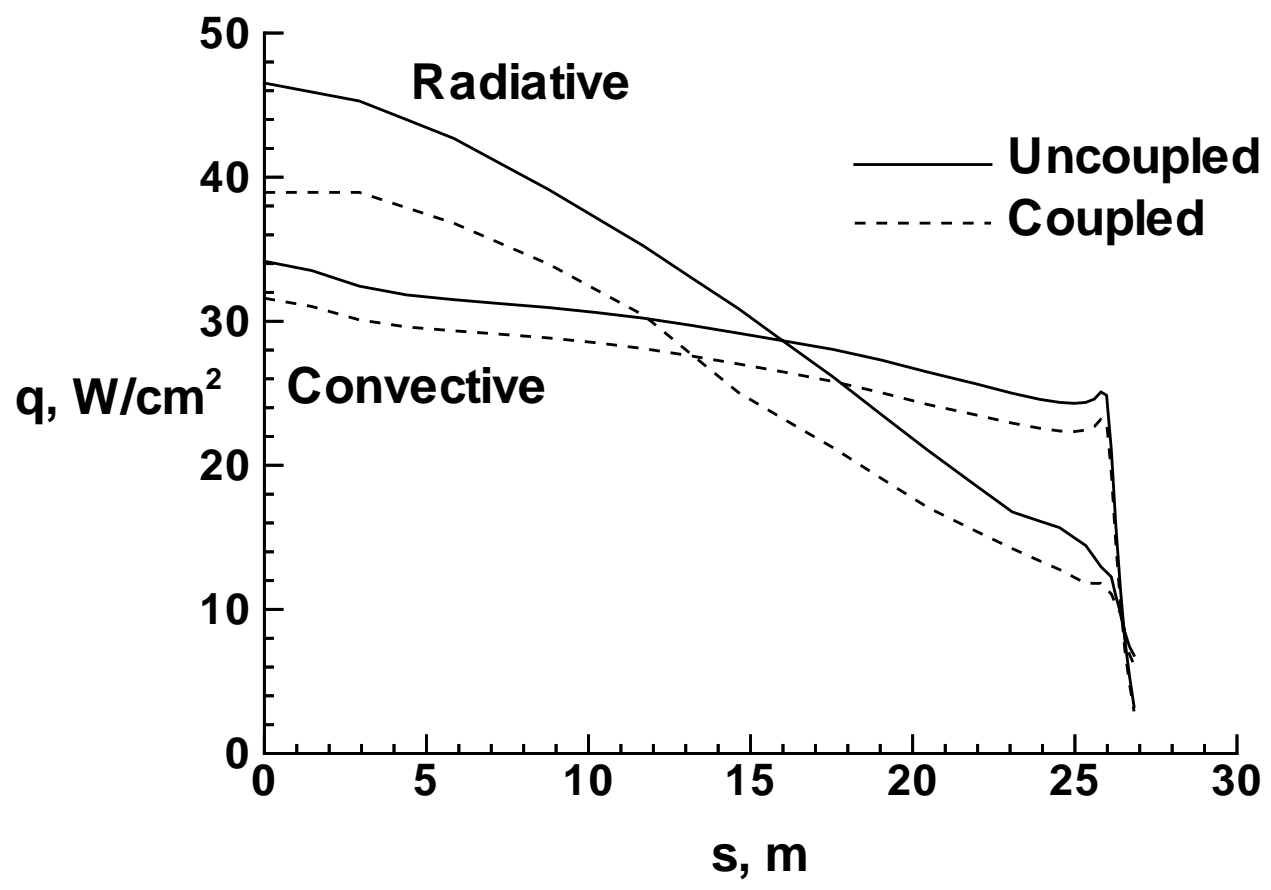

Figure 4: Effect of radiation coupling on surface heating for the lunar transfer vehicle. 


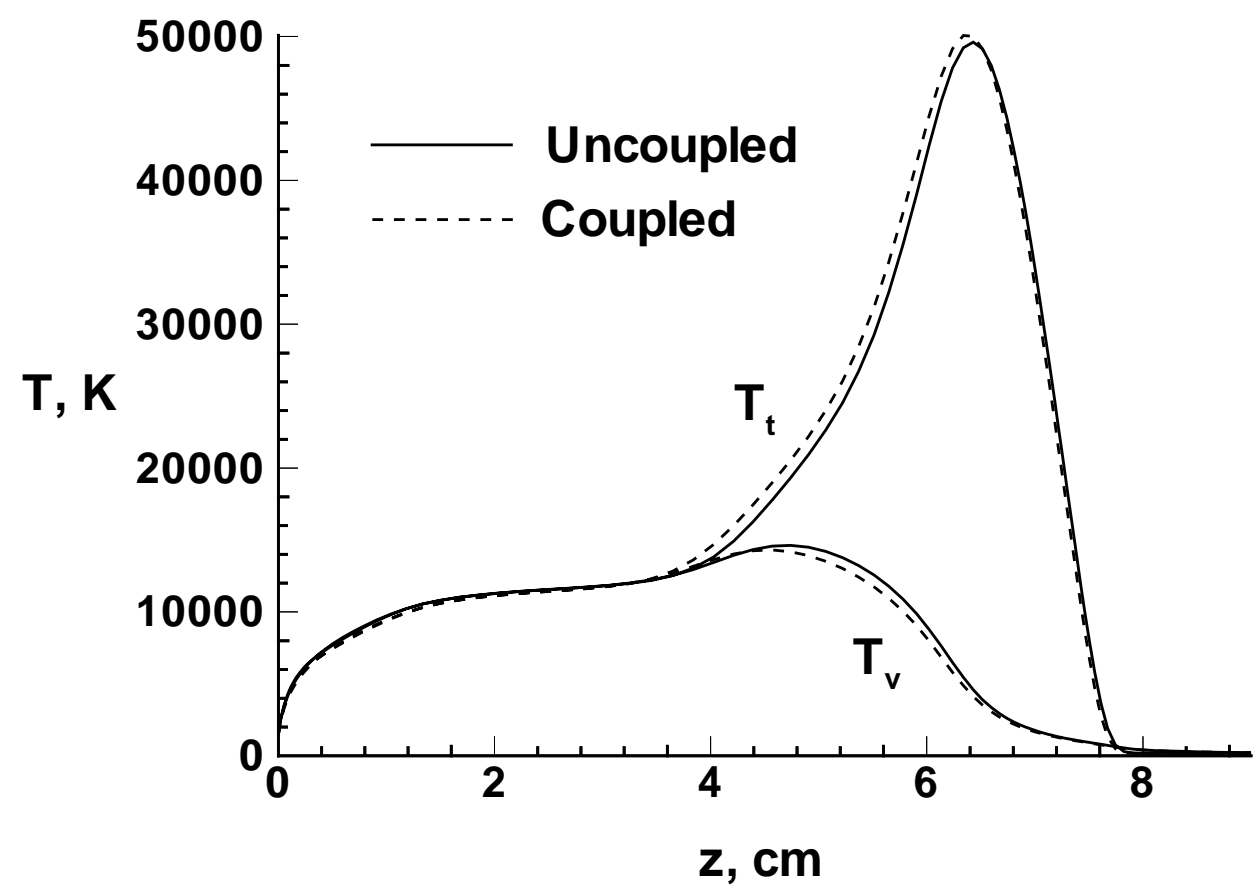

Figure 5: Effect of radiation coupling on the stagnation streamline temperature profiles for the Mars capsule return condition

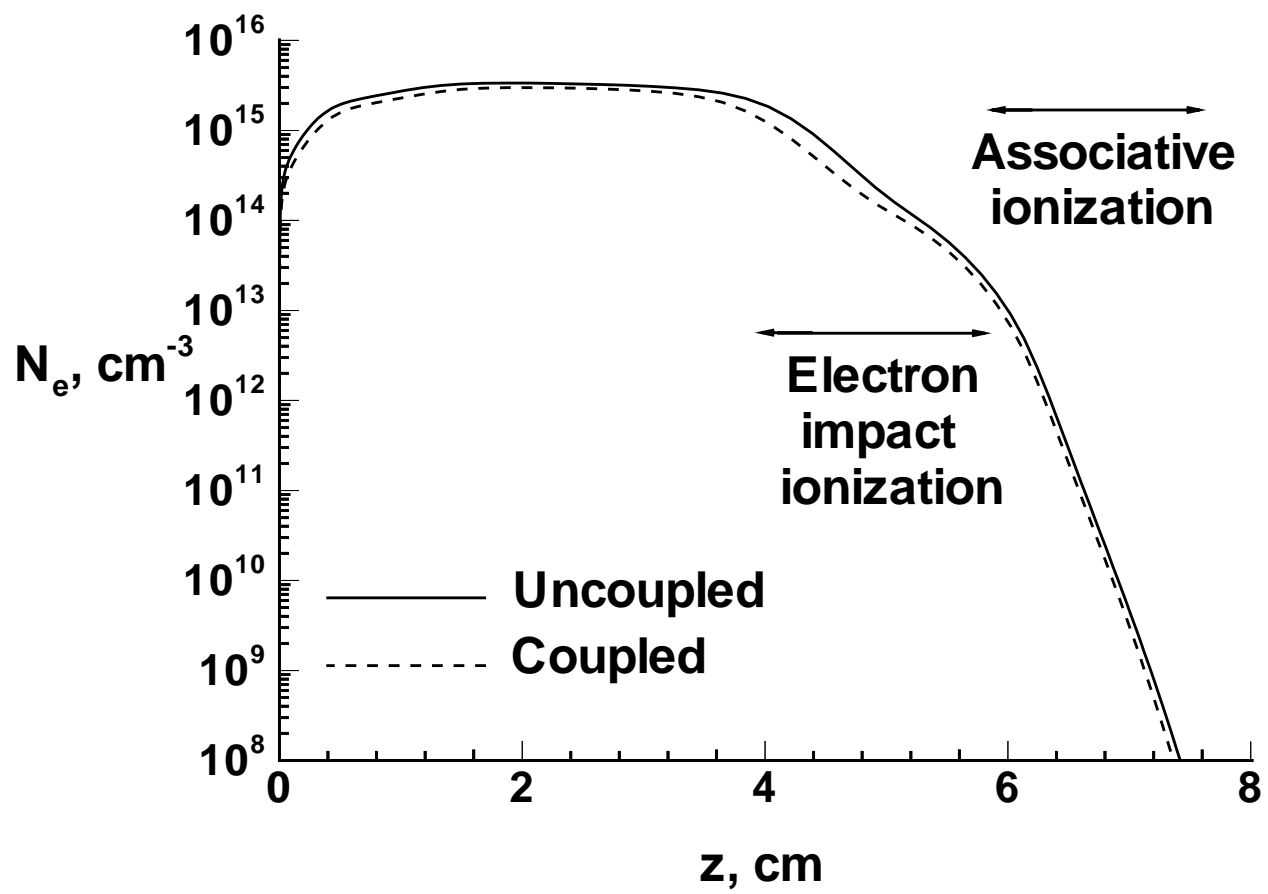

Figure 6: Effect of radiation coupling on stagnation streamline electron number density profiles for Mars capsule return condition. 


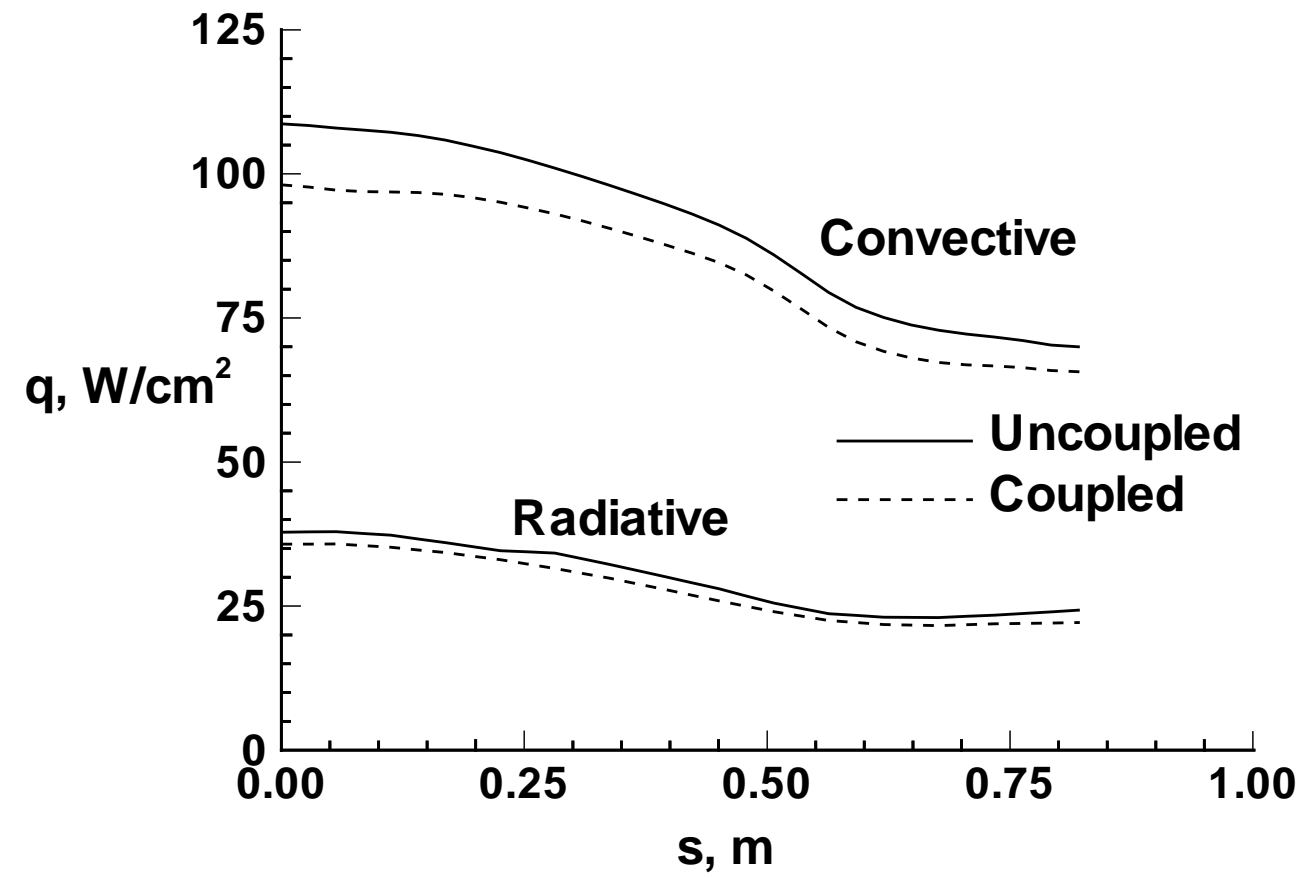

Figure 7: Effect of radiation coupling on surface heating for the Mars capsule return condition.

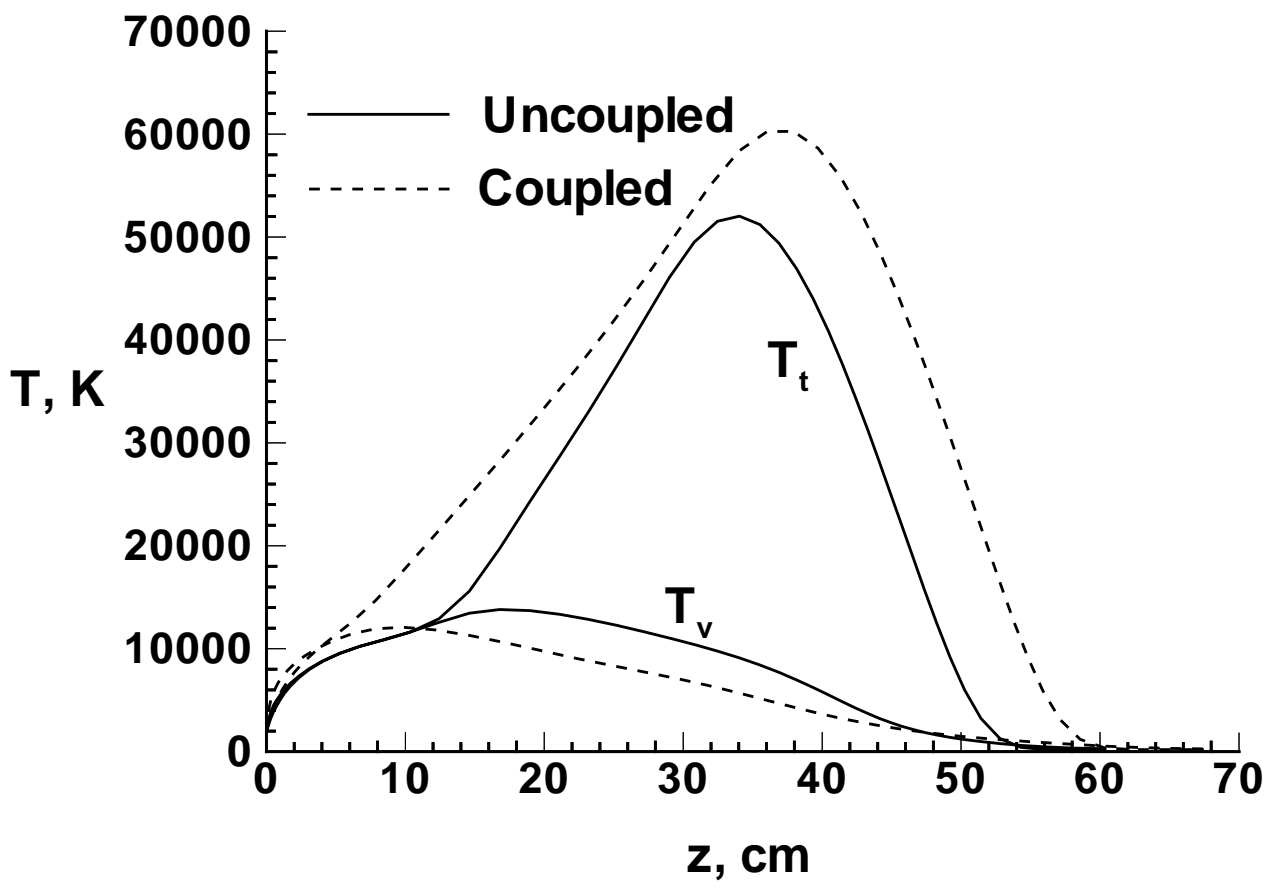

Figure 8: Effect of radiation coupling on the stagnation streamline temperature profiles for the Mars aerobrake return condition - Point 1. 


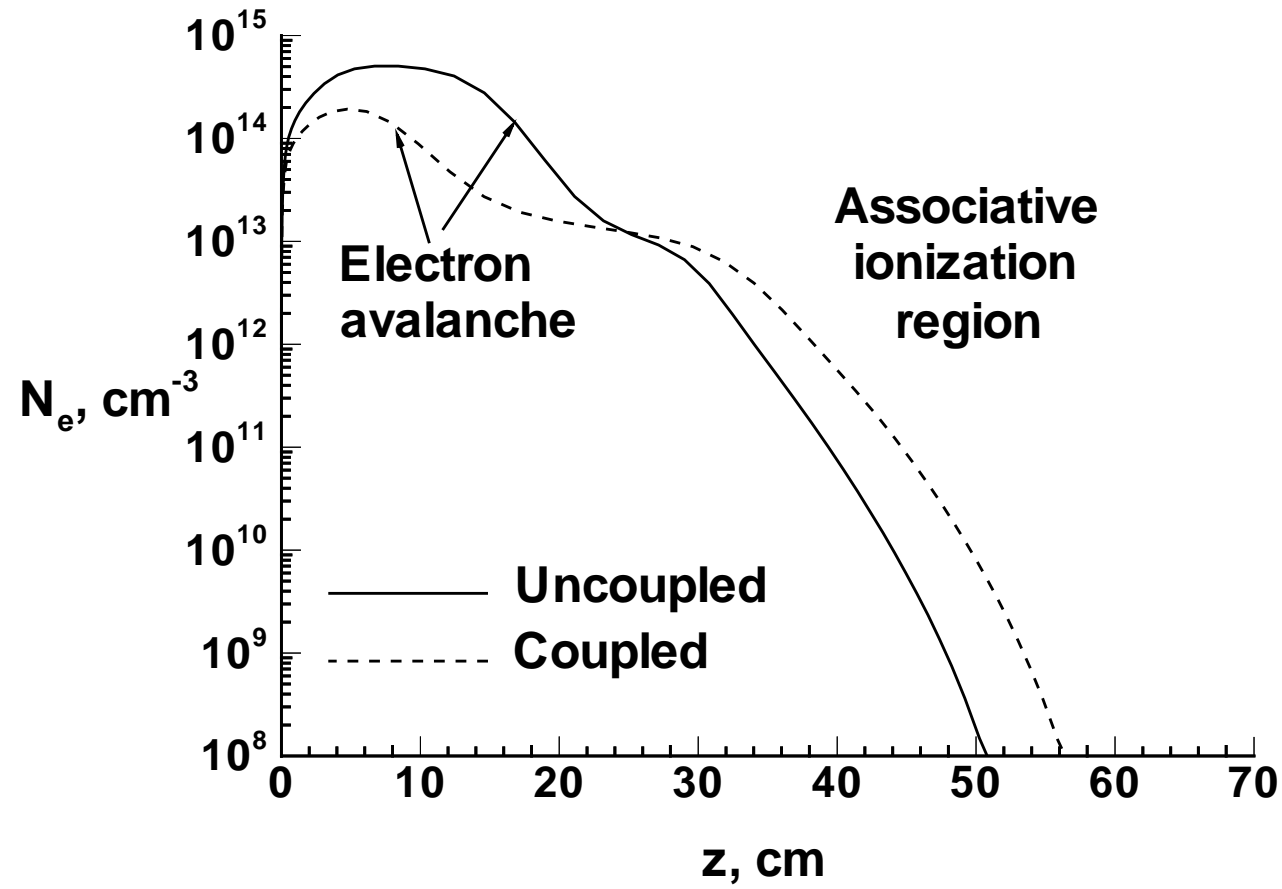

Figure 9: Effect of radiation coupling on electron density for the Mars aerobrake return condition - Point 1.

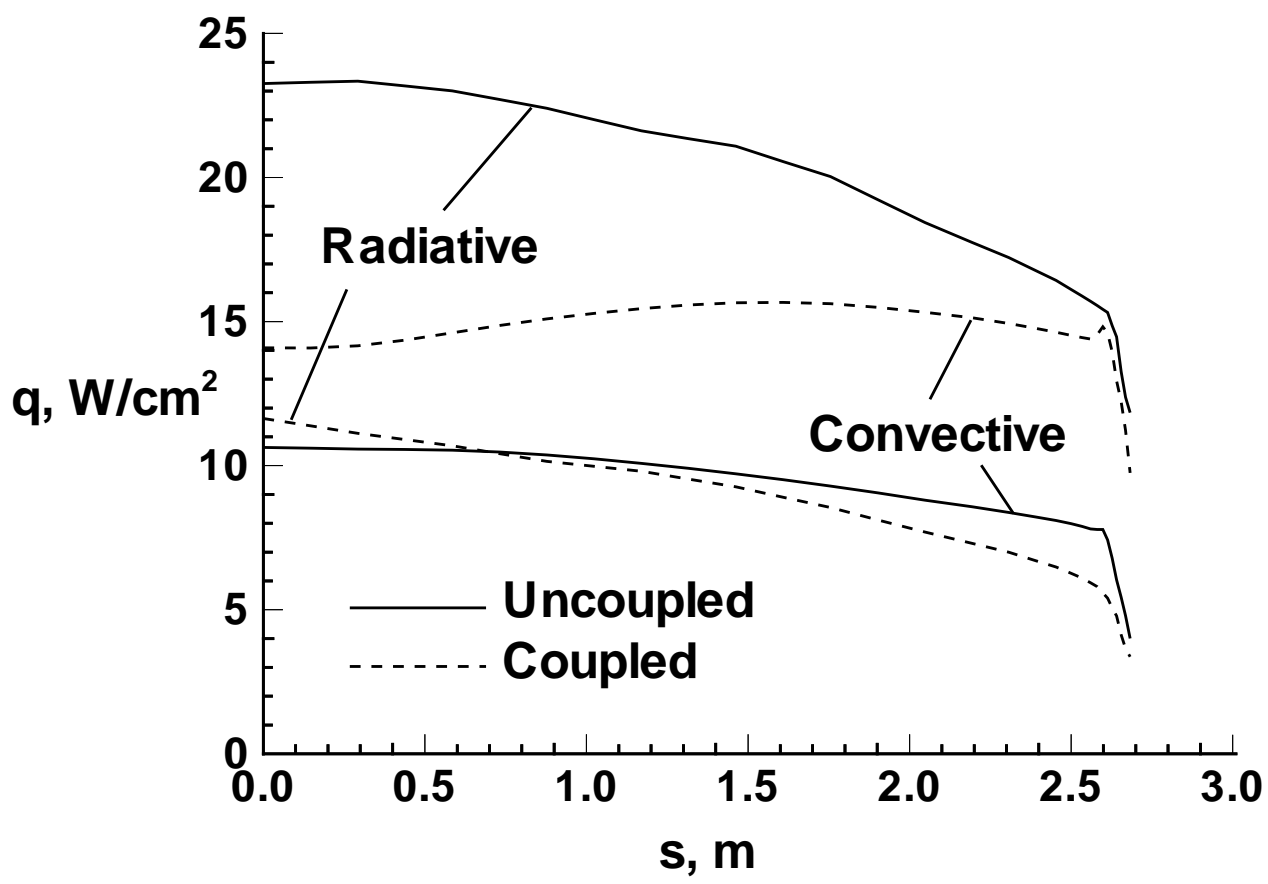

Figure 10: Effect of radiation coupling on surface heating for the Mars aerobrake return condition - Point 1. 


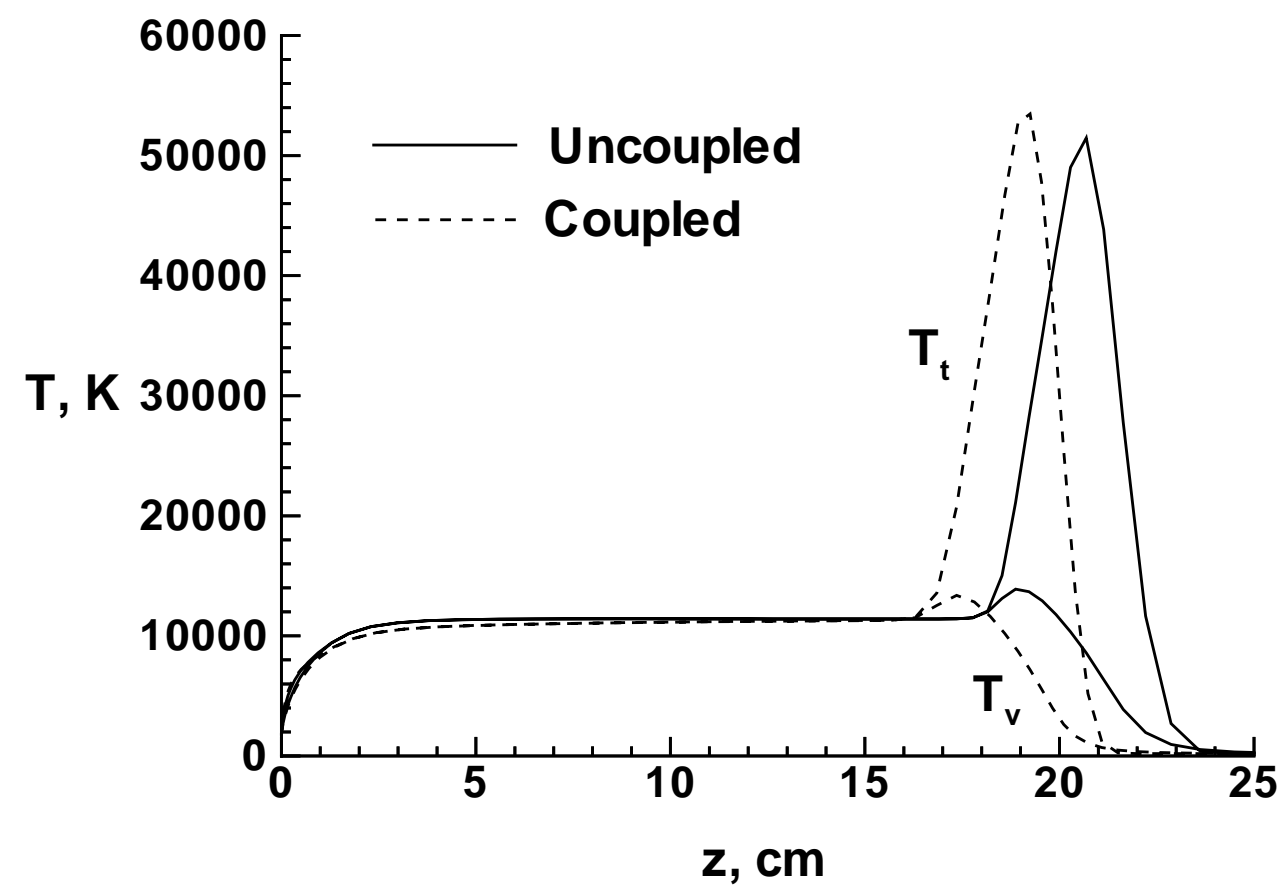

Figure 11: Effect of radiation coupling on the stagnation streamline temperature profiles for the Mars aerobrake return condition - Point 2

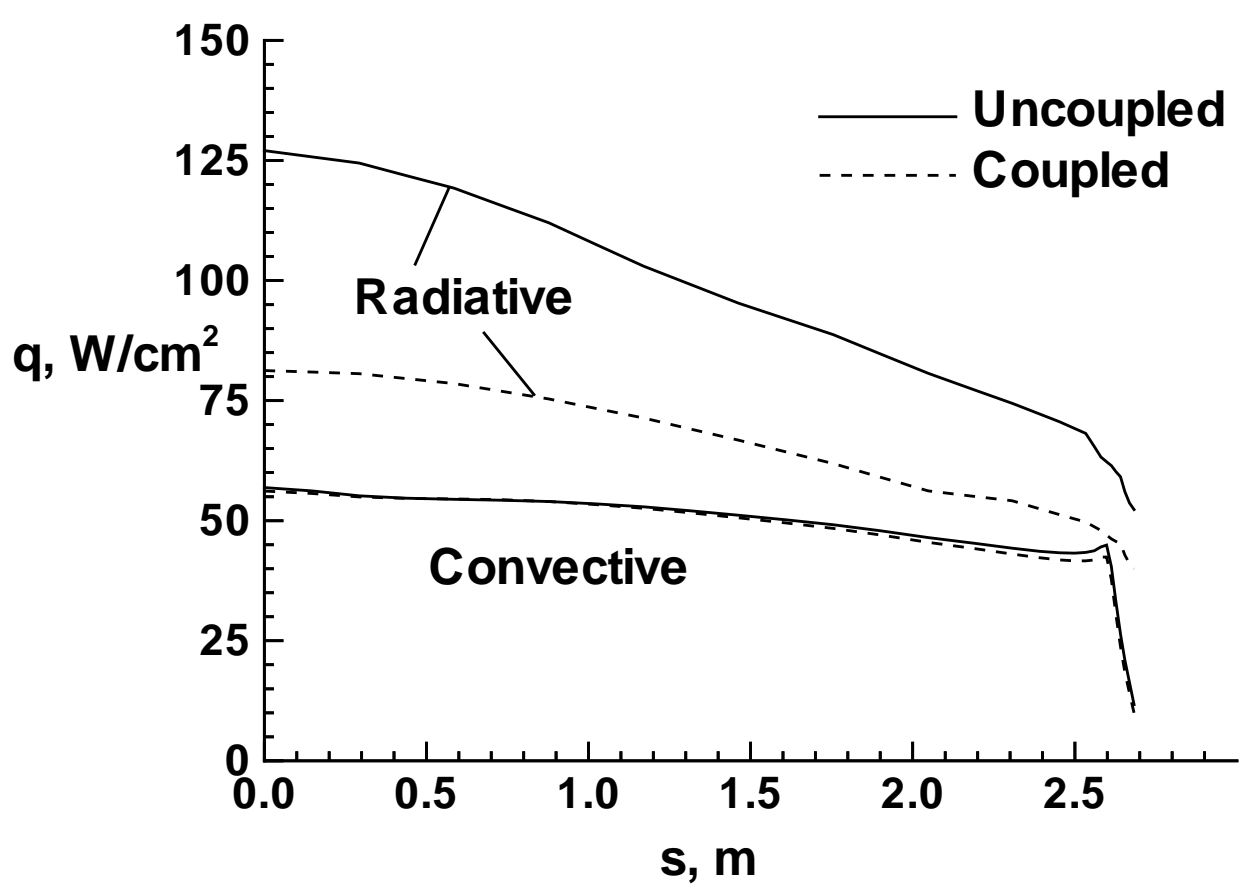

Figure 12: Effect of radiation coupling on surface heating for the Mars aerobrake return condition - Point 2. 


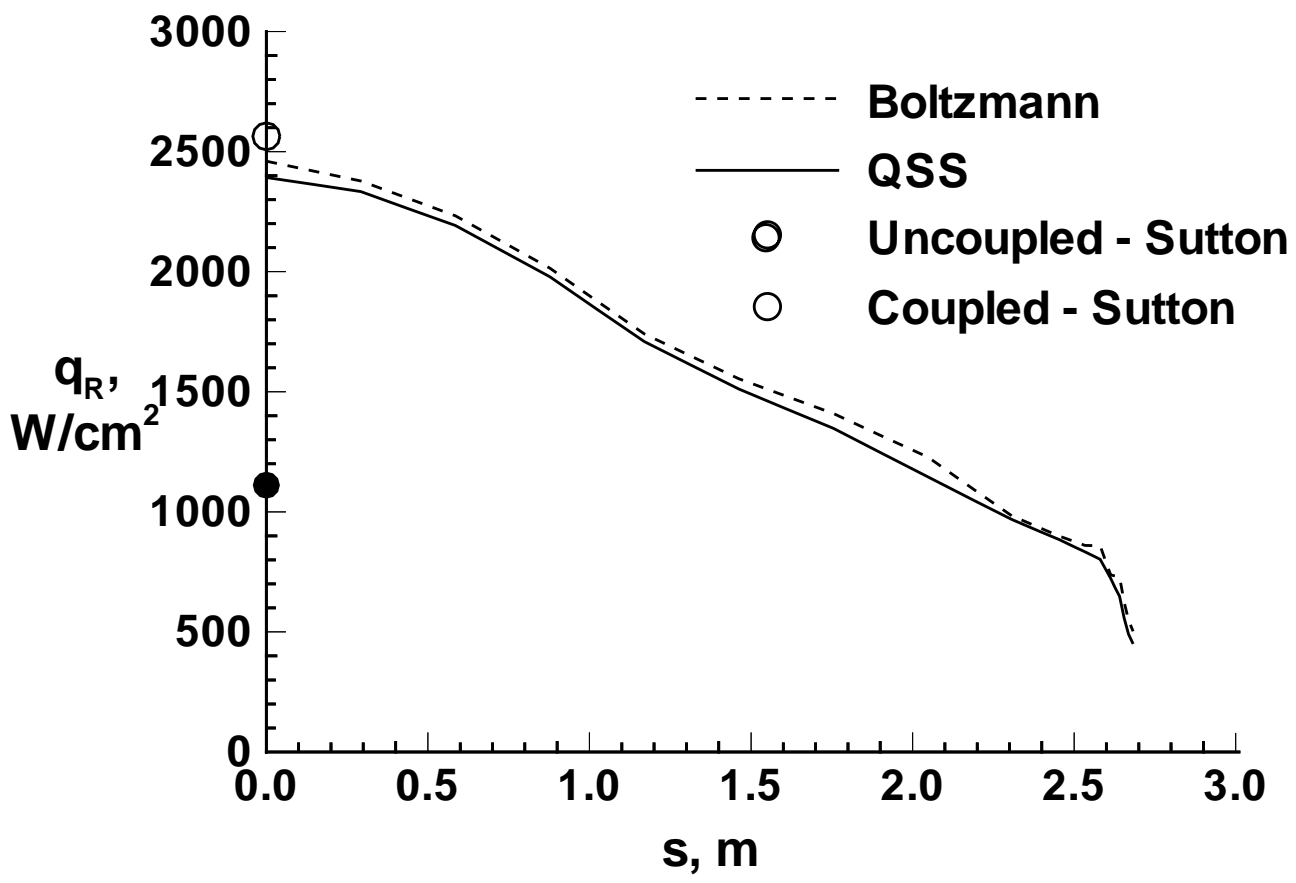

Figure 13: Wall radiative heating predictions for the Mars aerobrake return condition - Point 3. 


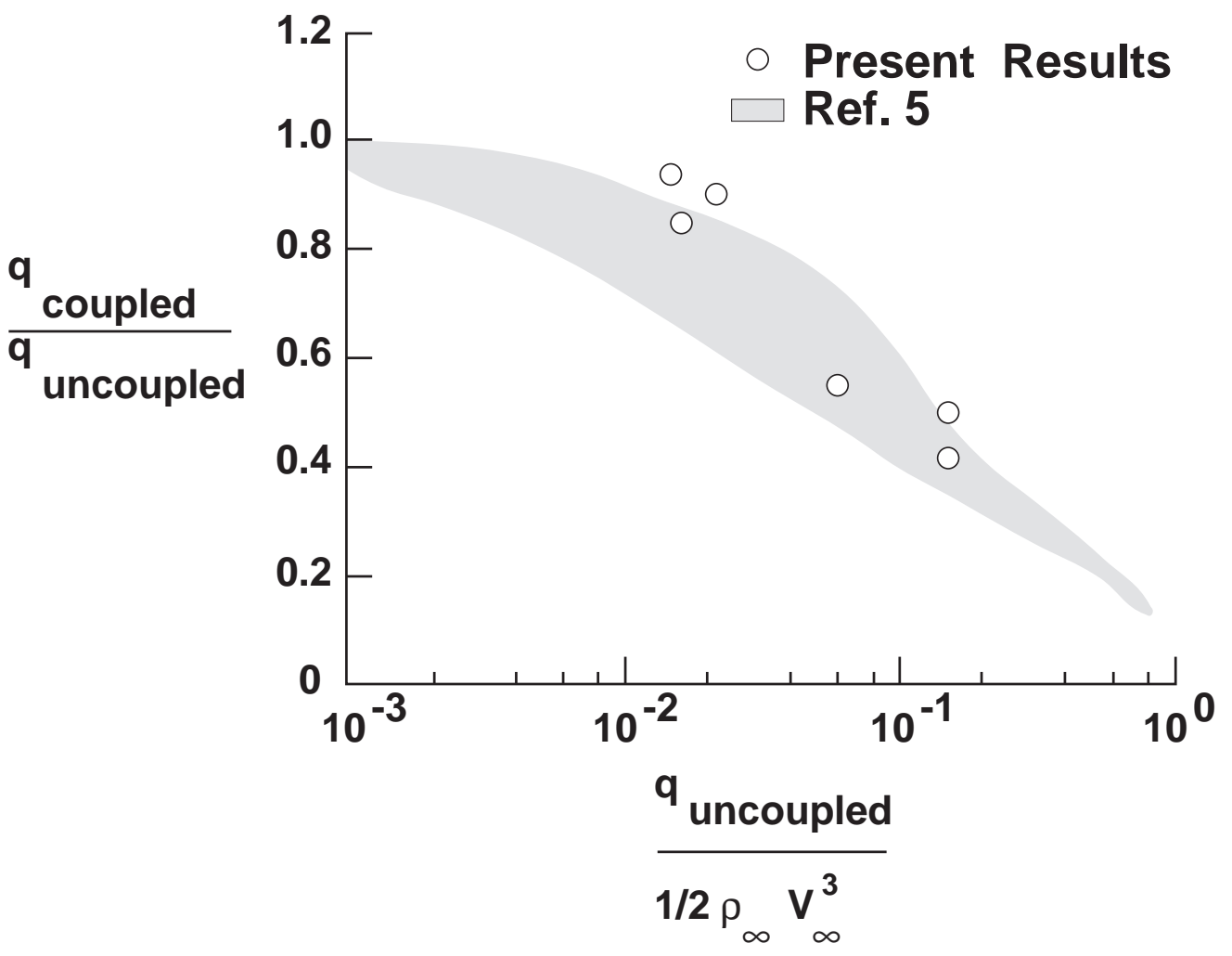

Figure 14: Cooling factor 